\title{
SOME FUNDAMENTAL THEORIES OF CRITICAL DISCOURSE ANALYSIS
}

Fariza Azkiya Ali

Department of English, Faculty of Teachers Training and Education, University of Lancang Kuning

farizaali70@gmail.com

\begin{abstract}
This paper study explains about some fundamental theories of Critical Discourse Analysis such as focus on dominance relations by elite groups and institutions as they are being ordained and the overt sociopolitical stance of discourse analysis. Context social structure: Situations of discursive interaction are similarly part or constitutive of social structure; for example, a press conference may be a typical repetition of organizations and media institutions. That is, "local" and more "global" contexts are closely related, and both exercise constraints on discourse. Personal and social cognition: Language users as social actors have both personal and social cognition: personal memories, knowledge and opinions, as well as those shared with members of the group or culture. Both types of cognition influence interaction 006 and discourse of individual members, whereas shared "social representations" govern the collective actions of a group.
\end{abstract}

Keyword: $C D A$, power, dominance, discourse

\section{INTRODUCTION}

This paper discusses some ideologies, goals and criteria of critical Discourse Analysis. CDA is a type of discourse analysis research that primarily studies the way social power abuse, dominance, and inequality are enacted, reproduced and resisted by text and talk in the social and political context. With such dissident research, critical discourse analysts take explicit position, and thus want to understand, expose, and ultimately resist social inequality.

DA provides a basic methodology to describes and analyze how the structure and content of the text encodes ideas and the relation among the ideas itself that are present in the text, systematically. (Hamuddin: 2012).

Critical research on discourse needs to satisfy a number of requirements in order to effectively realize its aims: first, as is often the case for more marginal research traditions. CDA research has to be "better" than other research in order to be accepted.

1) It focuses mostly on social problems and political issues, rather than on current paradigms and fashions. 
2) More exactly, CDA focuses on the ways discourse structures enact, confirm, legitimate, duplicate/challenge relations of power and dominance in society

\section{CONCEPTUAL AND THEORETICAL FRAMEWORKS}

Since CDA is not a specific way of research, it does not have a unitary theoretical framework. Within the aims mentioned above, there are many types of CDA, and these may be hypothetically and logically quite diverse. Critical analysis of conversation is very different from an analysis of news reports in the press or of lessons and teaching at school. Yet, given the mutual perspective and the general aims of CDA, we may also find overall conceptual and theoretical frameworks that are closely related.

As suggested, most kinds of CDA will ask questions about the way specific discourse structures are deployed in the reproduction of social dominance, whether they are part of a conversation or a news report or other kinds and situations. Thus, the typical vocabulary of many scholars in CDA will feature such notions as "power," “dominance," "hegemony," “ideology," “class," "gender," "social structure," "discourse and admission", and "social order," besides the more familiar discourse analytical notions. In this section, I focus on a number of basic concepts themselves, and thus devise a theoretical framework that critically relates discourse, cognition, and society.

This paper does not discuss the historical backgrounds and developments of critical perspectives in the study of language, discourse and communication. Nor does it provide a full bibliography of such work. Depending on the discipline, orientation, school or paradigm involved, these lines of development are traced back, if not as usual to Aristotle, then at least to the philosophers of the Enlightenment or, of course, to Marx, and more recently to the members of the Frankfurt School (Adorno, Benjamin and others) and its direct or indirect heirs in and after the 1960s, among whom Jürgen Habermas plays a primary role (Geuss, 1981; Jay, 1973; Slater, 1977). 
Another line of influence and development, also more or less (neo-) Marxist, is the one going back to Gramsci, and his followers in France and the UK, including most notably Stuart Hall and the other members of the Centre for Contemporary Cultural Studies (Corcoran, 1989; Hall, 1981). Likewise, first in France, later also in the UK and the USA, we can trace the influence of the work of Althusser (1971), Foucault (see, e.g., Foucault, 1980) and Pêcheux (1982), among others. Finally, we should emphasize the exemplary role of feminist scholarship in the critical approach to language and communication (for a bibliography, see Thorne et al., 1983).

\section{FUNDAMENTAL AND PURPOSE OF CDA}

Here, it refers to the speech patterns and how language, dialects, and acceptable statements are used in a community. Discourse as a subject of study looks at discourse among people who share the same speech conventions. Moreover, discourse refers to the linguistics of language use as a way of understanding interactions in a social context, specifically the analysis of occurring connected speech or written discourse, Dakowska (2001) in Hamuddin (2012).

The questions rose above about the purpose and the specific nature of CDA should be answered by a detailed technical discussion about the place of discourse analysis in contemporary scholarship and society. Such a discussion should specify, inter alia, the criteria that are characteristic of work in CDA. Instead, we shall simply, and perhaps naively, summarize such criteria by saying that in our opinion CDA should deal primarily with the discourse dimensions of power abuse and the injustice and inequality that result from it. Let us spell out some implications of such a lofty overall aim (see also Mey, 1985; O Barr, 1984: Steiner, 1985).

The focus on dominance and inequality suggests that, unlike other domains or approaches in discourse analysis, CDA does not primarily purpose to contribute to a specific discipline, paradigm, school or discourse theory. It is primarily interested and motivated by pressing social issues, which it hopes to better understand through discourse analysis. Theories, descriptions, methods and 
empirical work are chosen or elaborated as a function of their relevance for the realization of such a sociopolitical goal. Since serious social problems are naturally complex, this usually also means a multidisciplinary approach, in which distinctions between theory. description and application become less relevant. This focus on fundamental understanding of social problems such as dominance and inequality does not mean ignoring theoretical issues.

Critical discourse analysis is far from easy. In my opinion it is by far the toughest challenge in the discipline. As suggested above, it requires true multidisciplinary, and an account of intricate relationships between text, talk, social cognition, power, society and culture. Its adequacy criteria are not merely observational, descriptive or even explanatory (Fairclough, 1985). Ultimately, its success is measured by its effectiveness and relevance, that is, by its contribution to change. In that respect, modesty is mandatory: academic contributions may be marginal in processes of change, in which especially those who are directly involved, and their acts of resistance, are the really effective change agents

\section{Social and Cognition}

Whereas the organization of discourse access represents one of the crucial social dimensions of dominance, that is, who is allowed to say/write/hear/ read what to/from whom, where, when and how, we have stressed that modern power has a major cognitive dimension. Except in the various forms of military. police, judicial or male force, the exercise of power usually presupposes mind management, involving the influence of knowledge, beliefs, understanding, plans, attitudes, ideologies, norms and values. Ultimately, the management of modes of access is geared towards this access to the public mind, which we conceptualize in terms of social cognition.

Socially shared representations of societal arrangements, groups and relations, as well as mental operations such as interpretation, thinking and arguing, inferencing and learning, among others, together define what we understand by social cognition (Farr and Moscovici, 1984; Fiske and Taylor, 1991; Wyer and Srull, 1984). Discourse, communication and (other) forms of action and interaction are monitored by social cognition (Van Dijk, 1989a). 
The same is true for our understanding of social events or of social institutions and power relations. Hence social cognitions mediate between microand macrolevels of society, between discourse and action, between the individual and the group. Although embodied in the minds of individuals, social cognitions are social because they are shared and presupposed by group members, monitor social action and interaction, and because they underlie the social and cultural organization of society as a whole (Resnick et al., 1991).

We know very little about the structure and operations of the softer (or hotter) forms of social cognition, such as opinions, attitudes, ideologies, norms and values. We shall merely assume that these evaluative social representations also have a schematic form, featuring specific categories (as the schema men have about women, or whites have about blacks, may feature a category appearance: Van Dijk, 1987a). The contents of such schematically organized attitudes are formed by general, socially shared opinions, that is, by evaluative beliefs. The general norms and values that in turn underlie such beliefs may he further organized in more complex, abstract and basic ideologies, such as those about immigrants, freedom of the press, abortion or nuclear arms. For our purposes, therefore, ideologies are the fundamental social cognitions that reflect the basic aims, interests and values of groups.

They may (metaphorically and hence vaguely) be seen as the fundamental cognitive programmers or operating systems that organize and monitor the more specific social attitudes of groups and their members. What such ideologies look like exactly, and how they strategically control the development or change of attitudes, is as vet virtually unknown (see, however, e.g. Billig, 1982, 1991; Rosenberg, 1988: Windisch. 1985).

It is also increasingly accepted that concrete text production and interpretation are based on so-called models, that is, mental representations of experiences, events or situations, as well as the opinions we have about them (Johnson- Laird, 1983: Van Dijk, 1987b; Van Dijk and Kintsch, 1983). Thus, a newspaper report about (specific events in) the war in Bosnia is based on journalistic models of that war, and these models may in turn have been 
constructed during the interpretation of many source texts, e.g. of other media, key witnesses, or the press conferences of politicians. At the same time, such models are shaped by existing knowledge (about Yugoslavia, wars, ethnic conflict, etc.), and by more or less variable or shared general attitudes and ideologies.

Note that whereas knowledge, attitudes and ideologies are generalized representations that are socially shared, and hence characteristic of whole groups and cultures, specific models are-as such-unique, personal and contextualized: they define how one language user now produces or under-stands this specific text, even when large parts of such processes are not autobiographically but socially determined. In other words, models allow us to link the personal with the social, and hence individual actions and (other) discourses, as well as their interpretations, with the social order, and personal opinions and experiences with group attitudes and group relations, including those of power and dominance. Here we touch upon the core of critical discourse analysis: that is, a detailed description, explanation and critique of the ways dominant discourses (indirectly) influence such socially shared knowledge.

\section{Discourse and Admission}

We have suggested that one of the social resources on which power and dominance are based is the advantaged admission to discourse and communication. Admission is an interesting but also a rather vague analytical notion (Van Dijk, 1989b, 1993b). In our case it may mean that language users or communicators have more or less freedom in the use of special discourse genres or styles, or in the participation in specific communicative events and contexts. Thus, only politicians have admission to parliamentary debates and top managers to meetings in the board room. People may have more or less active or passive admission to communicative events, as is usually the case for journalists, professors or bosses when writing for, or speaking to, a more or less passive audience. Similarly, participants may have more or less control over the variable properties of the (course of) discourse and its conditions and consequences, such 
as their planning, setting, the presence of other participants, modes of participation, overall organization, turn-taking, agenda, topics or style.

An analysis of the various modes of discourse access reveals a rather surprising parallelism between social power and discourse access: the more discourse genres, contexts, participants, audience, scope and text characteristics they (may) actively control or influence, the more powerful social groups, institutions or elites are. Indeed, for each group, position or institution, we may spell out a discourse access profile. Thus, top business managers have exclusive admission to executive board meetings, in which the most powerful is usually associated with the chair, who also controls the agenda, speech acts (e.g. who may command whom), turn allocation (who is allowed to speak), decision-making, topics and other important and consequential dimensions of such institutional talk. At the same time, managers have admission to business reports and documents, or can afford to have those written for them; they have preferential admission to the news media, as well as to negotiations with top politicians and other top managers. Similar profiles may be sketched for presidents, prime ministers, political party leaders, newspaper editors, anchor(wo)men, judges, professors, doctors or police officers.

Many, prominent research with linguistic orientation mentions, the discourse of news report can be seen from two dimensions; the structure of news text and news production. In 1988, Teun van Dijk explain these dimensions in his book News as Discourse. The first dimension is the text, as this encodes values and ideologies that impact on and reflect the larger world. Many past studies in this area especially in linguistics orientation consider the news text from the vantage points of discourse structure or linguistic function, or according to its impact as ideology-bearing discourse. The second dimensions, news production that of the process including the norms and routines of the community of news practitioners. Therefore, this paper sees these two dimensions as inseparable part in the framework of this paper. It bounds explicitly to the approach design to analyze news, primarily as a type of text or discourse. 


\section{CONCLUSION}

There are many ways to do critical discourse analysis. Paradigms, philosophies, theories and methods may differ in these many approaches, and these may sometimes also be related to national differences, e.g. between French, German, British or American directions of research. Unfortunately, this is also one of the reasons why there has been much mutual neglect and ignorance among these different approaches. This means, among other things, that we presuppose a serious analysis of the very conditions and modalities of inequality, e.g. in terms of social power, dominance and their reproduction. In a critical study, such an analysis is not limited to a sociological or political-scientific account of dominance or patterns of access to social resources. Rather, positions and perspectives need to be chosen, for instance, against the power elites and in solidarity with dominated groups, as we have tried to illustrate in our analysis of the speech of Mr Fox in the British parliament. Such choices influence virtually all levels of theory and method.

\section{BIBLIOGRAPHY}

Hamuddin, B. (2012). A comparative study of politeness strategies in economic journals (Doctoral dissertation, University of Malaya).

Hamuddin, B., \& Noor, F. N. M. (2015, August). A Closer Look on Politeness Strategies in Malaysian Economic Journal. In 2nd INTERNATIONAL SEMINAR ON LINGUISTICS (p. 52).

Van Dijk, Teun A. 2015. Principle of Critical Discourse Analysis: Amsterdam. University of Amsterdam 